Rev. Elev. Méd. vét. Pays trop., 1978, 31 (3) : 341-352.

\title{
Inventaire parasitologique de l'Ethiopie : Helminthes des zébus adultes de la région de Kofélé (Arussi)
}

\author{
par M. GRABER $(*)$, R. P. DELAVENAY $(* *)$ et GEBRENEGUS TESFAMARIAN (***)
}

\begin{abstract}
RÉSUMÉ
Les auteurs dressent la liste des helminthes recueillis à l'autopsie d'un lot de zébus adultes originaires de la région de Kofélé (Sud de la Province de l'Arussi, Ethiopie).

Ils observent, dans le tractus digestif et dans le foie, la présence de nombreux Trématodes et Nématodes fréquemment associés entre eux. Il en résulte un polyparasitisme qui, dans 20 p. 100 des cas environ, s'avère particulièrement grave.

Par ailleurs, ils retrouvent, là encore, les deux zoonoses majeures si répandues en Ethiopie : la ladrerie à Cysticercus bovis avec un taux d'infestation de 14,6 p. 100 et l'echinococcose à Echinococcus polymorphus qui affecte plus de 50 p. 100 des animaux autopsiés et provoque parfois leur mort par cachexie.
\end{abstract}

Depuis une trentaine d'années, de nombreuses enquêtes effectuées en Ethiopie ont permis de se faire une idée assez précise de la nature et de l'importance du parasitisme des animaux domestiques.

Elles ont concerné d'abord l'Erythrée, les Provinces du Tigré, du Begemder, du Harrar, du Wolamo, du Shoa et, plus récemment, le Wollo, le Sidamo, le Gemu-Goffa, le Balé et le Harrar-Ogaden. Les résultats obtenus ont fait l'objet de plusieurs rapports $(1,6,8)$ et de diverses publications $(3,6,8)$.

Toutefois, ces recherches n'ont que peu intéressé la Province de l'Arussi (10) qui, de par sa position géographique et sa richesse en bétail, alimente l'abattoir d'Addis-Abéba dans le Shoa

(*) Chaire de Parasitologie, Ecole Nationale Vétérinaire de Lyon, Marcy l'Etolle, 69260 Charbonnières-lesBains.

(**) Mission Vétérinaire Française en Ethiopie, P. O. Box 1053, Addis-Abéba, Ethiopie.

(***) Veterinary Institute, P. O. Box 19, Debré-Zeit, Ethiopie. et l'abattoir industriel de Wondo-Genet (Shashamane) dans le Sidamo.

Ayant eu la possibilité, à la fin de l'année 1976, de procéder à l'autopsie complète d'un lot important de bovins provenant du sud de la Province, c'est-à-dire de la région de Kofélé, il a paru opportun de relater les observations faites à cette occasion.

\section{LE MILIEU}

L'Arussi est une petite province de $23500 \mathrm{~km}^{2}$ sise entre le Shoa, le Harrar et le Balé. Ses limites sont comprises entre la vallée du Rift, les lacs de Langano et de Zwai à l'Ouest, le cours supérieur de l'Awash au Nord, les montagnes du Harrar (Mont Gugu) à l'Est et la haute vallée de l'Ouadi Shébélé au Sud (Carte $n^{0} 1$ ).

C'est un vaste plateau d'altitude parsemé de pics dont certains dépassent $4100 \mathrm{~m}$ (Mont Cacca, Mont Badda, Mont Chillalo), plateau qui descend à $1200 \mathrm{~m}$ au voisinage des lacs de la vallée du Rift. 


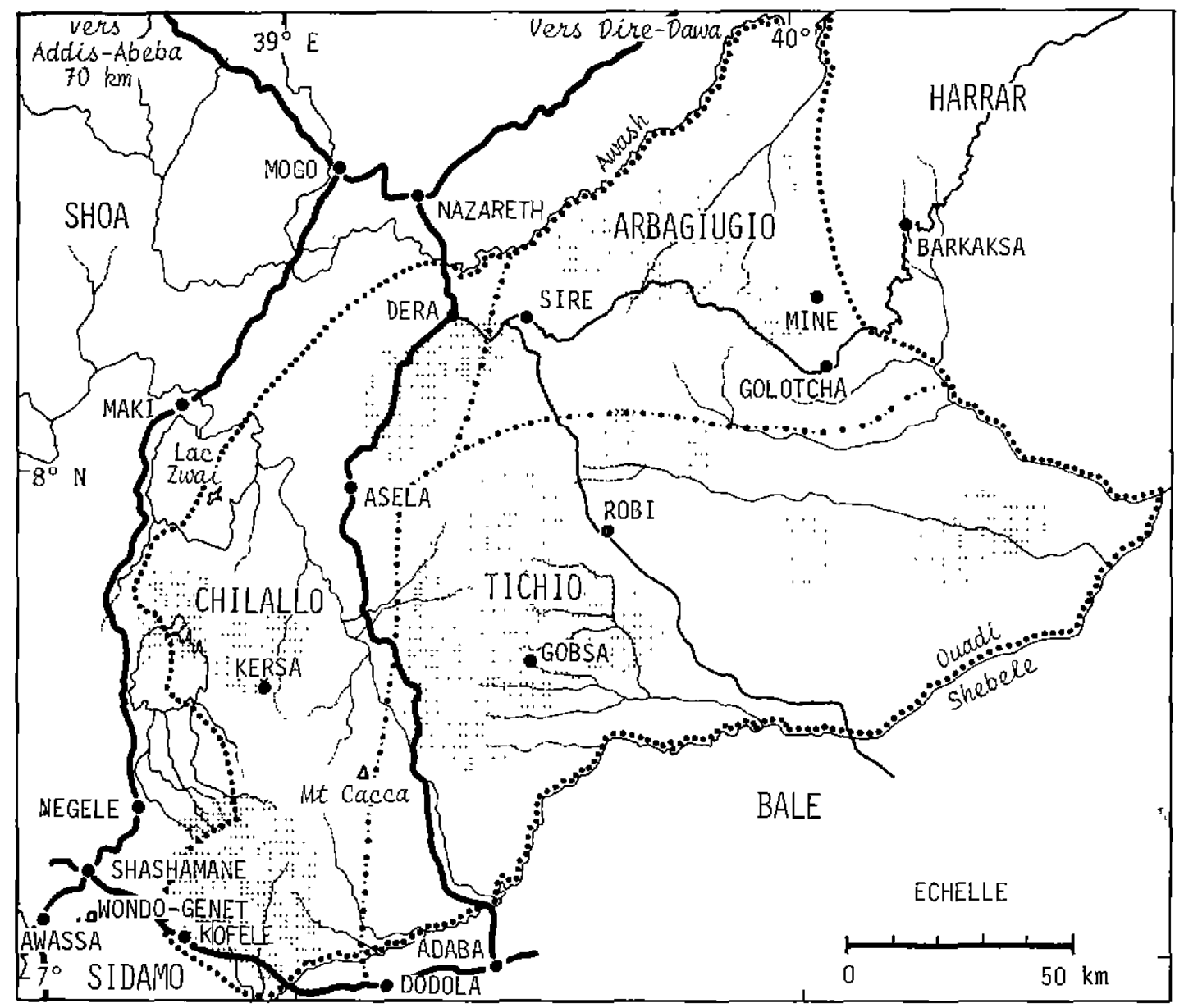

LEGENDE

PROVINCE DE L'ARUSSI

Limite de la province

Limite des districts

Routes principales
Routes secondaires

: : : $:$ :... Densitë de bëtail

TICHIO District
Peuplée d'environ 1,1 million d'habitants, la province de l'Arussi est une région agricole où l'élevage prime la culture. Les statistiques officielles dénombrent :

- 1119000 bovins

- 445000 moutons

- 192000 chèvres

- 104000 chevaux

- 100000 mulets et ânes.

Le bétail est surtout abondant dans les districts d'Arbagiugio, dans l'Ouest du district de Tichio et dans le sud du Chillalo, c'est-à-dire dans la région de Kofélé. C'est un élevage de type sédentaire: les mouvements de bétail se font, en saison sèche, des basses terres vers les hautes terres et sont donc fort restreints.
La région de Kofélé est située à $2680 \mathrm{~m}$ d'altitude à $7^{\circ} 4^{\prime}$ de latitude Nord et à $38^{\circ} 37^{\prime}$ de longitude est.

Les précipitations annuelles, sur une période de 11 ans (1966 à 1976) s'élèvent en moyenne à $1159 \mathrm{~mm}$, avec des périodes fortement bénéficiaires $(+10$ à +16 p. 100$)$ comme en 1967 , 1969 et 1970 et des périodes déficitaires ( -14 à - 16 p. 100) en 1973, 1974 et 1975.

La moyenne mensuelle des précipitations est la suivante :

- janvier : $29,1 \mathrm{~mm}$

- février : $74,5 \mathrm{~mm}$

- mars : $121,7 \mathrm{~mm}$

- avril : $144,8 \mathrm{~mm}$ 
_ mai : $93,4 \mathrm{~mm}$

— juin : $122,5 \mathrm{~mm}$

- juillet : $100,7 \mathrm{~mm}$

- août : 166,8 mm

- septembre : $139,9 \mathrm{~mm}$

- octobre : $87,6 \mathrm{~mm}$

- novembre : $57 \mathrm{~mm}$

- décembre : $21 \mathrm{~mm}$.

Il existe donc 2 saisons des pluies bien marquées :

- La première, la plus courte, va de mars à mai. Elle est provoquée par le déplacement d'un courant d'air humide de l'Océan indien en direction des zones de basse pression régnant sur le Soudan ;

-- La seconde, la plus longue, débute à la mi-juin et se termine en novembre. Elle est sous la dépendance des vents du Sud-Ouest soufflant des zones de haute pression du Sud Atlantique vers les zones de basse pression du Sahara, de l'Arabie et du Turkestan.

La saison sèche qui s'intercale entre les 2 saisons des pluies peut être, selon les années, plus ou moins longue : elle est, en général, de 3 mois (novembre à février) avec des extrêmes de un (1968) à 5 mois (novembre 1972 à avril 1973).

Les températures moyennes oscillent entre $12,1^{\circ} \mathrm{C}$ (novembre-décembre) et $14,4^{\circ} \mathrm{C}$ (marsavril). Sur 11 ans, eiles demeurent assez constantes, sauf en 1973 où elles ont dépassé $18^{\circ} \mathrm{C}$ durant 2 mois (mars-avril).

La région de Kofélé qui fait partie de la zone climatique du « Dega » (Terre froide) jouit donc d'un climat tempéré qui assure la repousse constante des pâturages, ce qui est particulièrement propice à l'élevage.

\section{MATÉRIEL ET MÉTHODE}

Cette enquête, effectuée en novembre-décembre 1976, a concerné un lot d'animaux achetés sur les marchés de Kofélé 2 mois auparavant et stockés sur la concession de la «Quarantaine " à Awassa (Sidamo).

Ce sont typiquement des zébus abyssins mâles, non castrés, pesant de 145 à $310 \mathrm{~kg}$, en moyenne $220 \mathrm{~kg}$ pour 85 animaux.

Il a été procédé à plusieurs séries d'opérations :

- sur 148 zébus, à des examens coprosco- piques selon la méthode multivalente d'enrichissement par sédimentation (6) ;

- sur 85 d'entre eux, à des coprocultures (méthode de Brumpt) et à des autopsies faites selon les techniques habituelles (5).

Les Trématodes et les Cestodes de l'appareil digestif ont été colorés au carmin chlorhydrique et montés au baume du Canada. Des coupes sériées médio-sagittales ont été pratiquées sur pius de 800 Paramphistomes (soit 1,3 p. $100 \mathrm{du}$ matériel récolté).

Les Nématodes, ainsi que les Cysticerques musculaires, ont été, avant examen entre lames et lamelle, éclaircis au lactophénol.

Les Echinocoques ont été systématiquement ouverts, de façon à apprécier leur degré de maturité.

\section{ESPẼCES PARASITES RENCONTRÉES}

Ont été identifiés :

1. Dans l'esophage

- Gongylonema pulchrum Molin, 1857.

2. Dans la panse et dans le duodénum, des Trématodes appartenant à 2 espèces différentes

2.1. Cotylophoron cotylophorum Fischieder, 1901.

Caractérisé essentiellement (Pl. 1, fig. 1 et 2) par l'existence d'une ventouse génitale saillante, bien visible, d'un bulbe œsophagien particulièrement volumineux, de 2 testicules disposés en diagonale et d'un acétabulum ( = ventouse postérieure) s'ouvrant ventralement (*).

La présence de ce parasite, fréquemment rencontré chez les ruminants domestiques et sauvages des zones soudano-guinéennes d'Afrique (Zaïre, Empire Centrafricain, Tchad, Nigéria) est formellement confirmée en Ethiopie (I). Dans ce pays, Cotylophoron cotylophorum

(*) L'identification des Paramphistomidés, encore actuellement, est basée sur le système de NASMARK, tel qu'il a été décrit dans le texte original de l'auteur (9) et dans de nombreuses autres publications (12) qui donnent la liste des caractères anatomiques permettant l'identıfication. Pour plus de détails, nous renvoyons à ces publications. 
Planche $n^{0}$ 1. - Cotylophoron cotylophorum.

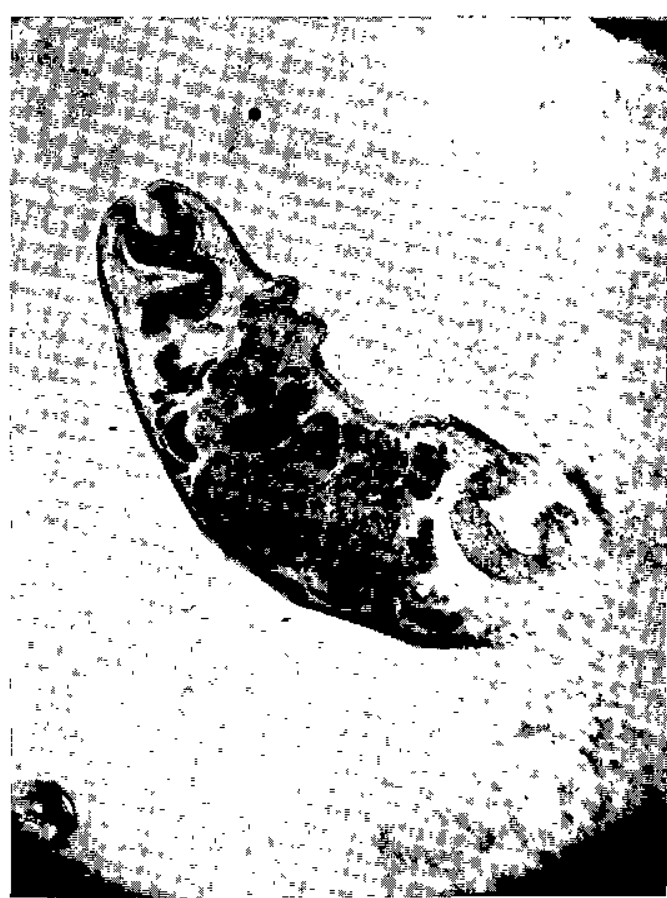

Fig. $n^{0}$ 1. - Vue générale $(\times 3,2)$.

n'affecte pas seulement les ruminants domestiques, mais également certains ruminants sauvages, comme l'indique une enquête menée récemment dans le parc national de l'Awash (II) : ce Trématode a, en effet, été recueilli à 2 reprises dans la panse d'un Nyala (Tragelaphus buxtoni) et celle d'un Bushbuck (Tragelaphus sp.).

\subsection{Paramphistomum daubneyi Dinnik, 1962.}

Paramphistomidé conique mesurant 5 à $10 \mathrm{~mm}$ de long sur 2 à $5 \mathrm{~mm}$ de large en avant de l'acétabulum. La cuticule de la face ventrale du corps présente des séries parallèles de plis transversaux. Le pore génital, légèrement en saillie, est situé à 1,5-1,8 $\mathrm{mm}$ de l'extrémité antérieure (Pl. 2, fig. 1).

L'acétabulum (Pl. 3, fig. 2) subterminal est de type Paramphistomum. La musculature circulaire externe et dorsale est divisée en 2 parties, 1(D. e. c. 1) et 2(D. e. c. 2), la partie 2 étant composée de 20 à 25 faisceaux bien individualisés et régulièrement espacés.

La musculature du pharynx, c'est-à-dire la partie antérieure du tube digestif (autrefois, la ventouse orale) comprend (P1. 3, fig. 1) :

- une couche circulaire interne (i. c. 1)

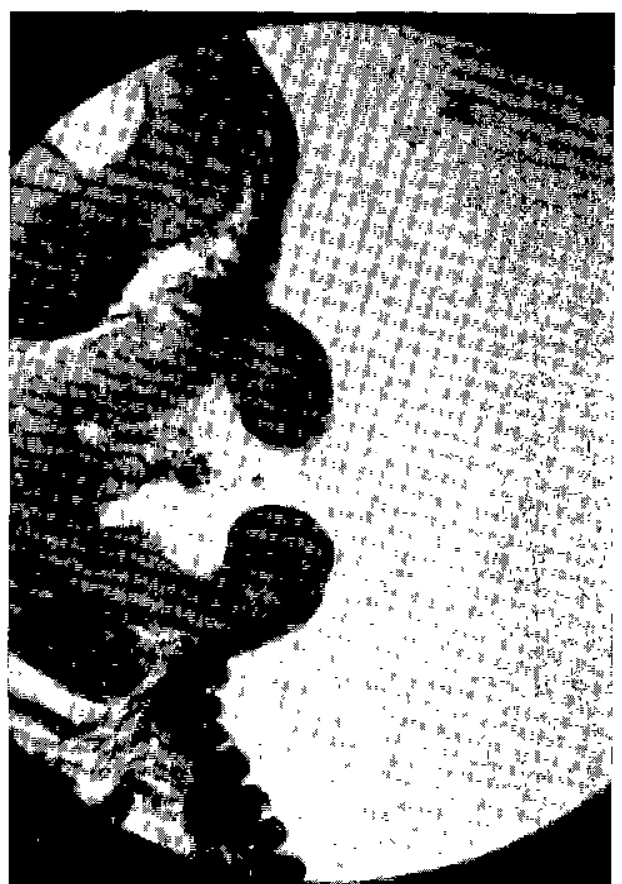

Fig. $n^{\circ} 2,-$ Ventouse génitale (détails).

faite de faisceaux serrés disposés en une seule rangée sur toute la hauteur de la paroi et dont la taille va en augmentant de haut en bas ;

- une couche longitudinale interne (i. 1. 1) formée de fibres de petite taille dirigées vers l'intérieur ;

- une couche circulaire médiane (m. c. 1) peu développée ;

- une couche circulaire externe (e. c. 1) présentant les caractères de la couche circulaire interne ;

- une couche longitudinale externe (e. 1. 1) de faible importance.

L'œsophage qui fait suite au pharynx est dépourvu de toute formation bulbaire.

Les caecums intestinaux se terminent de chaque côté de l'acétabulum.

Les testicules (Pl. 2, fig. 2) sont situés l'un derrière l'autre au milieu du corps. Ils sont divisés en de nombreux lobes (15 à 25 selon les exemplaires).

L'atrium génital (Pl. 2, fig. 3) est de type Microbothrium. Bien délimité, il est entouré de chaque côté d'un pli dermique circulaire muni d'un sphincter puissant. La papille génitale porte à sa surface de petits sphincters. 
Planche $\mathrm{n}^{\circ}$ II. - Paramphistomum daubneyi

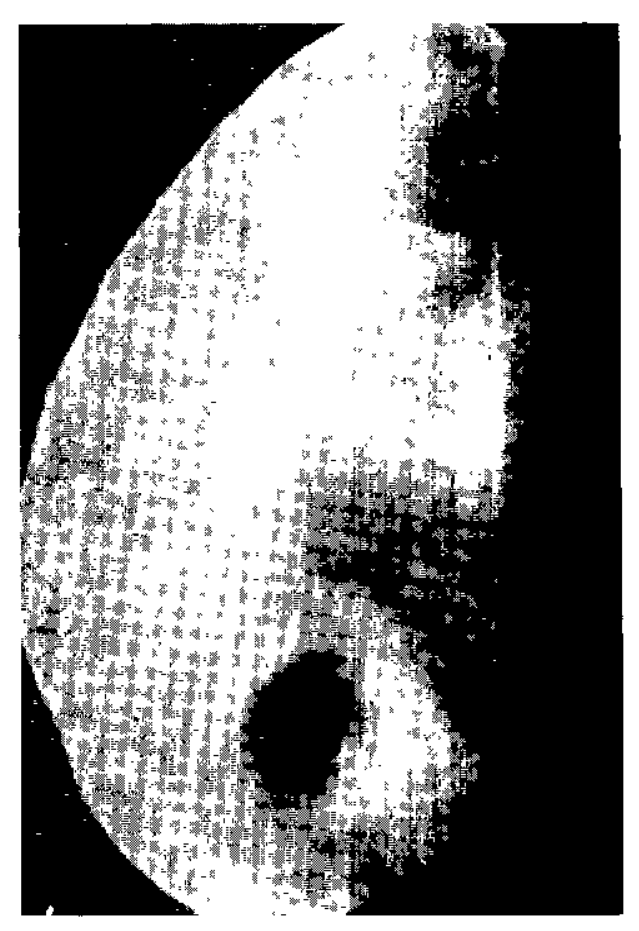

Fig. $n^{\circ} 1$, - Vue générale $(\times 2)$.

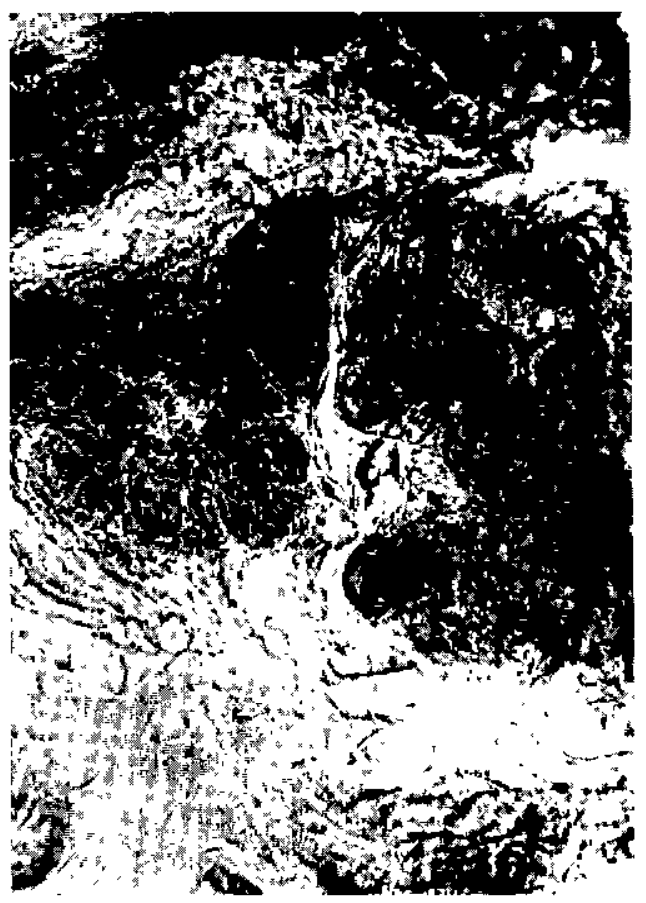

Fig. $\square^{\circ}$ 2. - Testicules $(\times 70)$.

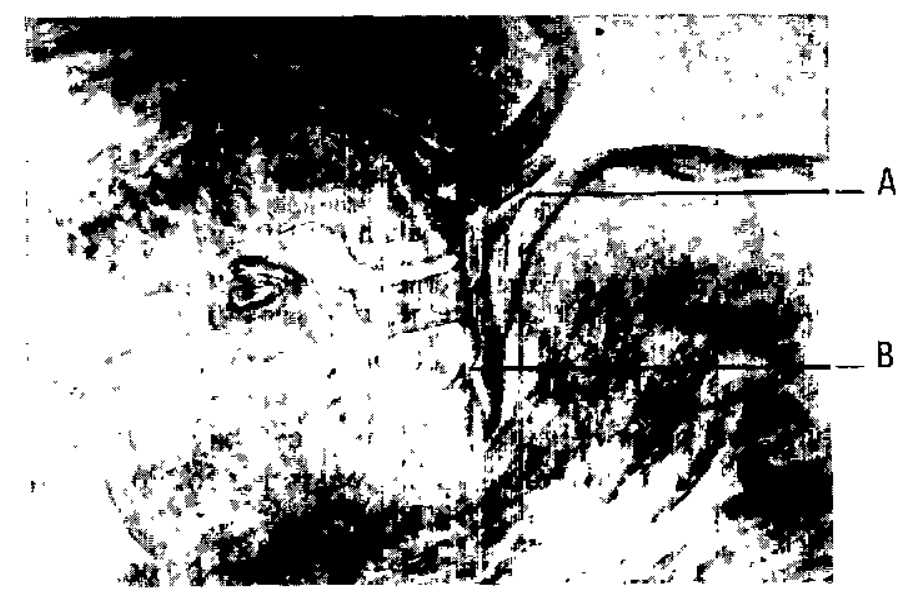

Fig. $n^{\circ} 3$. - Alrium génital $(\times 300)$.

A. Papille génitale ;

B. Pli dermique et sphincter; 
Planche $n^{\circ}$ III. - Paramphistomum daubneyi.

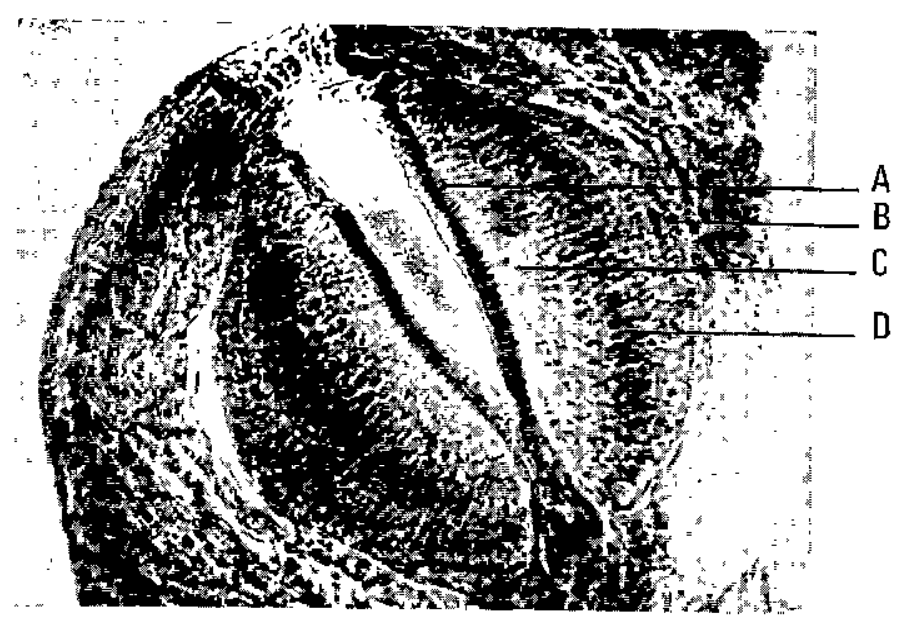

Fig. $n^{\circ} 1 .-$ - Pharynx ( $\left.\times 110\right)$.

A. Couche circulaire interne (i. c. 1.).

B. Couche circulaire externe (e. c. 1.);

C. Couche longitudinale interne (i. 1. 1.);

D. Couche circularre médiane (m. c. 1.).

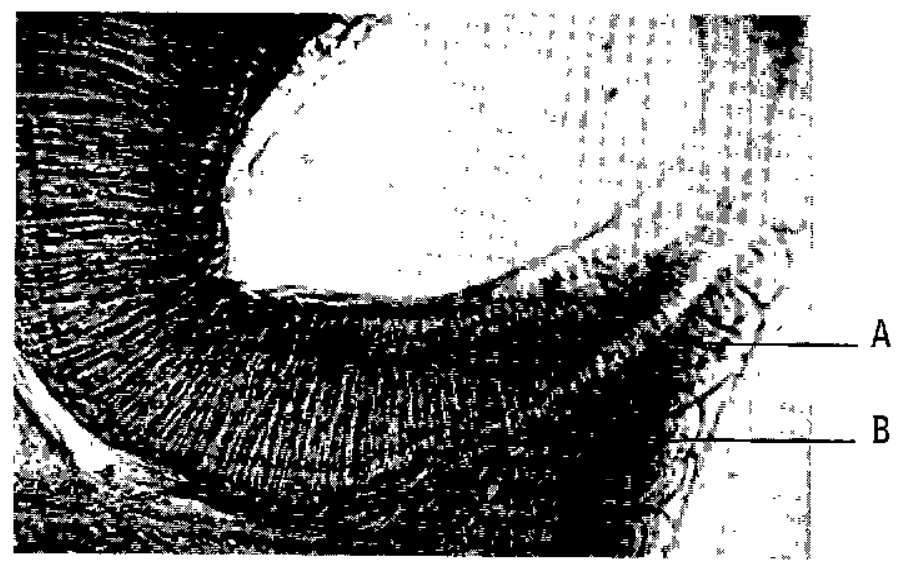

Fig. n० 2, - Acétabulum ( $\times 110)$

A. Musculature circulaire externe et dorsale - Partie 1 .

B. Musculature circulaire externe et dorsale - Partie 2.

Les œufs grisâtres, allongés, à pôles inégaux mesurent 120-150 $\mu \times 60-72 \mu$ (Moyenne, $137 \mu \times 68 \mu$ ).

L'aspect général du parasite, les caractères de l'acétabulum, du pharynx, des testicules, de l'atrium génital, les dimensions des ceufs correspondent à la description originale de Paramphistomum daubneyi faite par DINNIK (4) et permettent de différencier ce Trématode d'autres espèces voisines existant en Ethiopie, notamment Paramphistomum sukari et Paramphistomum microbothrium.
Dans ce pays, Paramphistomum daubneyi n'est pas un inconnu, puisqu'il a déjà été identifié :

- chez des bovins, dans le Wollo (6), à Kembolcha et à Dessié ;

- chez des moutons, dans le Balé, à Dinscho.

Il est probable que ce Paramphistomidé colonise - comme au Kenya (4) - les hautes terres du Dega qui sont particulièrement favorables au développement et au maintien de son hôte intermédiaire, la limnée tronquée (Galba truncatula). 


\section{Dans la caillette}

- Haemonchus contortus Rudolphi, 1803.

\section{Dans l'intestin grêle}

\subsection{Des Cestodes: Moniezia expansa Rudolphi,} 1810.

C'est, en Ethiopie, un parasite banal du mouton, de la chèvre, du dromadaire et du zébu $(1,6)$. Le Téniasis des bovins, qui touche surtout les jeunes veaux, est sporadique et irrégulièrement réparti.

\subsection{Des Nématodes appartenant aux espèces} suivantes

- Bunostomum phlebotomum Railliet, 1900.

- Cooperia punctata Von Linstow, 1907.

- Cooperia pectinata Ransom, 1907.

- Trichostrongylus sp $(*)$.

- Nematodirus sp. $\left({ }^{*}\right)$.

Les 4 premières espèces sont largement répandues sur tout le plateau éthiopien $(1,6,7)$. Les Nematodirus, facilement reconnaissables grâce à leurs cufs de grande taille, sont beaucoup plus rares et leur présence n'a été signalée, jusqu'à présent, que chez quelques bovins et ovins des montagnes du Harrar (7).

\section{Dans le cæcum et le gros intestin}

- Oesophagostomum (Bosicola) radiatum $\mathrm{Ru}-$ dolphi, 1803.

\section{Dans le foie et les canaux biliaires}

\subsection{Des Trématodes}

- Fasciola hepatica Linné, 1758.

- Fasciola gigantica Cobbold, 1855.

6.2. Un Cestode: Echinococcus polymorphus, Diesing, forme larvaire d'un Taenia des carnivores domestiques et sauvages, Echinococcus granulosus Batsch, 1786.

\section{Dans le poumon}

- Echinococcus polymorphus, Diesing.

\section{Dans la rate}

- Echinococcus polymorphus, Diesing.
9. Dans le cour

- Echinococcus polymorphus, Diesing.

- Cysticercus bovis, forme larvaire d'un Taenia de l'homme, Taenia saginata Gœze, 1782.

10. Dans le mésentère

- Cysticercus tenuicollis, Rudolphi, forme larvaire de Taenia hydatigena, Pallas, 1766, parasite intestinal des carnivores domestiques et sauvages.

\section{Oeil}

- Thelazia rhodesii Desmarest, 1827. Ce spiruridé que l'on trouve dans toute l'Ethiopie (1) provoque des conjonctivites vermineuses fréquemment compliquées de kératite. Aussi, revêtent-elles souvent un caractère de haute gravité.

\section{COMMENTAIRES}

Près de 93 p. 100 des zébus de Kofélé (137 sur 148) sont porteurs de parasites.

1. Du point de vue de la patbologie, 2 affections méritent de retenir l'attention :

1.1. Les rrématodoses de l'appareil digestif et du foie

1.1.1. La distomatose hépato-biliaire, selon les statistiques de l'abattoir de Wondo-Genet (Sidamo) $\left({ }^{*}\right)$, touche plus de 60 p. 100 des animaux de boucherie originaires de Kofélé et sacrifiés dans cet abattoir industriel.

Comme il a été dit plus haut, les parasites en cause sont Fasciola hepatica et Fasciola gigantica. L'autopsie de 75 zébus reconnus atteints à l'examen coproscopique a permis d'établir la répartition par espèces :

- nombre d'animaux porteurs de Fasciola hepatica: 43 , soit 57,4 p. 100 ;

- nombre d'animaux porteurs de Fasciola gigantica: 14 , soit 18,6 p. 100 ;

— infestations mixtes : 18 , soit 24 p. 100 .

Dans le cas des infestations mixtes, les Fasciola hepatica sont 3 fois plus nombreuses que les

${ }^{*}$ ( ) Dr GOUALO, Directeur de l'abattoir, Communication personnelle.

(") Sur examens coproscopiques et coprocultures. 
Fasciola gigantica. Celles-ci sont, la plupart du temps, immatures et de petite taille, ce qui confirme les observations faites précédemment dans le Wollo (6).

L'existence de ces 2 Distomes, souvent associès entre eux, n'est pas étonnante, car on sait maintenant qu'en Ethiopie, l'hôte intermédiaire de Fasciola hepatica, Galba truncatula, colonise les zones humides du plateau éthiopien à partir de $1200 \mathrm{~m}$ et que Limnea natalensis, le vecteur de Fasciola gigantica, est capable de se développer dans certaines collections d'eau jusqu'à $2200 \mathrm{~m}$ d'altitude $(2,8)$. Dans l'Arussi, les mouvements de bétail des basses terres riches en Limnea natalensis vers les hautes terres où Galba truncatula est l'espèce dominante explique pourquoi l'on retrouve les deux Trématodes, seuls ou associés.

L'importance des lésions est extrêmement variable. Il s'agit :

- dans 46,6 p. 100 des cas, d'une distomatose massive avec cirrhose et cholangite généralisées ;

- dans 25,3 p. 100 des cas, d'une distomatose moyenne, localisée surtout au lobe gauche du foie, avec importante cholangite des gros canaux de la face postérieure.

- dans 28,1 p. 100 des cas, d'une distomatose légère se traduisant par une cholangite modérée (origine : Fasciola hepatica, 2/3 ; Fasciola gigantica, 1/3). Souvent, il se forme, sur le trajet des plus gros canaux biliaires, une poche plus ou moins volumineuse, faisant saillie à la surface de l'organe, poche à parois fortement épaissies et où s'accumulent les Fascioles. Le passage de la bile ne se fait pratiquement plus ou très mal. Il y a, en quelque sorte, formation d'un véritable kyste enchassé dans le parenchyme hépatique.

Le nombre de douves recueillies à l'ouverture des canaux est sans aucune mesure avec l'étendue des lésions : 150 au maximum, lors de distomatose massive, 20 à 50 dans les cas de distomatose moyenne.

Le petit nombre de parasites rencontrés, leur état de maturité différent, l'irrégularité des pontes explique pourquoi, à l'examen coproscopique, le pourcentage d'animaux atteints est bien inférieur à celui que l'on observe à l'abattoir : 35 p. 100 (52 zébus sur un total de 148) contre 60 p. 100.

Un second examen, pratiqué $48 \mathrm{~h}$ après le premier, permet de faire remonter le taux à 45 p. 100. Mais, de toutes les façons, la méthode d'enrichissement par sédimentation n'arrive à déceler que 75 à 85 p. 100 des animaux infestés.

Le rôle pathogène de Fasciola hepatica et de Fasciola gigantica est bien connu : ces Trématodes sont hématophages, ils absorbent une assez grande quantité de vitamines, ils modifient le métabolisme du fer et gênent la digestion.

La distomatose hépato-biliaire qui, chez les bovins, prend rarement une allure aiguë a, sur le plan économique, des conséquences graves. Outre la mortalité qu'elle peut occasionner chez des animaux en mauvais état et mal nourris, elle provoque chez les jeunes des retards de croissance et, chez les animaux destinés à la boucherie, des pertes de poids et une diminution de la qualité de la viande.

1.1.2. Les Paramphistomes de la panse et du duodénum affectent 83 p. 100 des zébus de la région de Kofélé (123 sur 148 examinés en coproscopie).

Le nombre de parasites recueillis à l'autopsie de chaque animal varie dans des proportions sensibles : de 150 à plus de 10000 .

On sait que, dans un premier temps, les Paramphistomes envahissent la sous-muqueuse $\mathrm{du}$ duodénum où ils demeurent 4 à 7 semaines, puis ils effectuent une migration rétrograde qui les amène dans la panse, parfois dans d'autres réservoirs gastriques, le réseau notamment. Ils y achèvent leur croissance. Pour Paramphistomum daubneyi, la durée de la phase prépatente est d'environ 92 jours (4).

Sur coupe, 80 p. 100 des parasites examinés avaient atteint leur maturité sexuelle. Deux fois seulement (sur 85 autopsies), la présence de très jeunes Paramphistomes a été observée dans la sous-muqueuse du duodénum, ce qui, compte tenu de la durée de la phase prépatente, situe la période d'infestation au début du mois de septembre.

Grâce à leur ventouse postéricure, les Paramphistomes s'accrochent aux papilles de la panse. Celles-ci subissent de fortes érosions, s'atrophient et, finalement, il se forme des plages dénudées dont l'étendue varie en fonction de l'importance du parasitisme. Ces lésions s'accompagnent de troubles digestifs divers, bien connus des éleveurs éthiopiens : anorexie, arumination, ballonnement, indigestion. Le rôle pathogène des Paramphistomes n'est donc nullement négligeable. 


\subsection{Les « strongyloses» gastro-intestinales}

Sur 85 animaux autopsiés, 57 d'entre eux étaient porteurs de Nématodes de la caillette et de l'intestin se répartissant ainsi :

- Oesophagostomum : 12, soit 21 p. 100.

- Bunostomum : 3, soit 5,2 p. 100.

- Haemonchus : 22, soit 38,5 p. 100.

- Cooperia: 21 , soit 36,8 p. 100.

- Trichastrongylus sp. : 11, soit 19,2 p. 100.

- Nematodirus sp. : 2, soit 3,5 p. 100.

Les espèces dominantes sont donc Cooperia et Haemonchus suivies d'Oesophagostomum et de Trichostrongylus. Ce type de parasitisme est très classique chez les zébus de toute l'Afrique tropicale.

Dans 25 p. 100 des cas, les Nématodes sont associés entre eux.

Ils ne sont jamais en très grand nombre, ce qui réduit d'autant leur rôle pathogène (6). Toutefois, dans cette classe d'âge, Bosicola radiatum présente un réel danger, surtout lorsque les lésions d'œsophagostomose nodulaire sont importantes et s'étendent sur pratiquement toute la longueur de l'intestin, ce qui - fort heureusement - n'a pas été observé dans le lot d'animaux autopsiés.

Quelques larves inhibées L4 de Cooperia et d'Haemonchus ont également été isolées.

1.3. "Strongles », $\left({ }^{*}\right)$ Fasciola et Paramphistomes sont associés entre eux dans 56 p. 100 des cas (84 sur 148).

Toutefois, les associations ne sont pas également pathogènes et il leur faut atteindre un certain seuil pour qu'elles le deviennent, seuil qui correspond à des infestations fortes ou massives. Ceux-ci, compte tenu de diverses observations antérieures (6) que confirme entièrement le résultat des autopsies effectuées à Awassa, peuvent être chiffrés ainsi :

- Infestations massives (plus de 2500 aufs $(* *)$ au gramme de matière fécale) : 4,7 p. 100 des animaux examinés en coproscopie.

- Infestations fortes (de 1000 à 2500 cufs au gramme de matière fécale) : 16,2 p. 100 .

(*) Haemonchus, Bosicola, Cooperia, Bunostomum et Trichostrongylus.

(**) Eufs de Fasciola, de Paramphistomes et de "Strongles».
On peut donc considérer que, sur l'ensemble du lot ayant fait l'objet de cette étude, 1 animal sur 5 est atteint d'un polyparasitisme déjà grave, dommageable pour sa santé.

\section{Du point de vue sanitaire, 2 zoonoses s'avèrent particulièrement importantes}

\subsection{La cysticercose à Cysticercus bovis.}

Seuls, les cœurs des 85 bovins autopsiés ont pu être examinés $(* * *)$. Quatorze d'entre eux, soit 16,4 p. 100 présentaient des vésicules ladres en nombre variable (de 1 à 9) à la surface de l'organe ou en profondeur.

Dans tous les cas, il s'agissait de cysticerques inermes appartenant à l'espèce Cysticercus bovis. Aucun cysticerque armé n'a été rencontré.

Le tiers à peine des vésicules avait subi la dégénérescence caséo-calcaire, avec cysticerques morts, partiellement calcifiés.

Les autres renfermaient un parasite bien vivant capable de se transformer chez l'hôte définitif, l'homme, en Taenia saginata. Aussi, les risques d'infestation, à partir d'animaux de boucherie parasités, sont-ils, dans cette région de l'Ethiopie, considérables.

\subsection{L'echinococcose d̀ Echinococcus poly- morphus.}

Sur 85 autopsies, le taux d'infestation est de 54 p. 100 , ce qui est supérieur au pourcentage établi par PERSSON et PERSSON (10) à l'abattoir d'Asella, la capitale de l'Arussi (44 p. 100).

Les kystes sont :

- soit localisés à un seul organe

- Poumon : 20 (43,5 p. 100$)$

- Foie : 2 (4,4 p. 100)

- Rate : 1 (2,1 p. 100) ;

- soit localisés à plusieurs organes

- Foie et poumon : $19(41,4$ p. 100$)$

- Poumon et rate : $1(2,1$ p. 100$)$

- Cour, Poumon et foie : $1(2,1$ p. 100$)$

- Foie, Poumon et Rate : 2 (4,4 p. 100).

(***) Ce pourcentage eût étć vraisemblablement supérieur si les carcasses avaient pu être mises en menus morceaux, ce qu'il n'a pas été possible de faire, faute de temps. 
Certains sont anciens, de petite taille et en grande partie calcifiés : 15 p. 100 lors de localisation pulmonaire, 25 p. 100 lors de localisation mixte poumon-foie.

Il arrive souvent que l'on trouve dans le foie des echinocoques calcifiés et, dans le poumon, des echinocoques normaux ou encore dans le foie des echinocoques normaux et, dans le poumon, en même temps, des échinocoques calcifiés et des échinocoques normaux.

D'une façon générale, dans cette région de l'Ethiopie, les localisations uniques et les localisations à plusieurs organes sont de fréquence égale, les cas d'échinococcose pulmonaire sont beaucoup plus nombreux que les cas d'échinococcose hépatique et la dégénérescence caséocalcaire touche surtout les kystes présents dans le foie.

Le nombre d'hydatides isolés sur chaque animal varie dans des proportions sensibles:

$$
\begin{aligned}
& -1 \text { à } 5,41,9 \text { p. } 100 \\
& -5 \text { à } 10,28 \text { p. } 100 \\
& -10 \text { à } 15,9,3 \text { p. } 100 \\
& -15 \text { à } 20,6,9 \text { p. } 100 \\
& -20 \text { à } 30,13,9 \text { p. } 100 .
\end{aligned}
$$

Lors d'infestation légère (moins de 10 parasites), les kystes sont, la plupart du temps, bien tolérés par leurs hôtes. Au-delà, surtout lorsqu'ils sont volumineux, ils sont susceptibles de provoquer des troubles sérieux :

- D'origine hépatique, avec perte de l'appétit, arumination et diarrhée.

- D'origine pulmonaire, avec disparition du murmure vésiculaire, matité, diminution des bruits du cœur, toux et dyspnée que l'on met en évidence en faisant courir l'animal sur une certaine distance.

L'examen clinique a permis de déceler 6 cas d'échinococcose pulmonaire typique et, chaque fois, l'autopsie a confirmé qu'il existait dans le poumon 15 à 19 kystes de toute taille $(1$ à $10 \mathrm{~cm}$ ). Le parenchyme compris entre les vésicules est fortement comprimé. Il s'atrophie peu à peu et prend une coloration grisâtre qui correspond à des zones de sclérose. Parfois, il disparaît complètement.

L'animal maigrit et cet amaigrissement peut être brutal aboutissant à ce que l'on nomme la « Cachexie hydatique ». C'est ce que nous avons observé sur un animal porteur de 18 kystes pulmonaires volumineux : en une semaine, il a perdu $45 \mathrm{~kg}$, alors que le reste du troupeau, entretenu dans les mêmes conditions, voyait son poids augmenter ou, tout au moins, se maintenir.

L'échinococcose, dans cette région de l'Ethiopie, pose donc un triple problème :

- Economique, en raison des saisies qu'elle entraîne, notamment des foies fort prisés par certains Ethiopiens qui les mangent crus. Les poumons n'étant pas consommés par la population sont laissés à la libre disposition des chiens, ce qui assure une large dissémination du parasite.

- De pathologie, puisque un nombre non négligeable d'animaux adultes présentent des signes cliniques d'échinococcose et que la maladie peut évoluer progressivement vers la cachexie et la mort.

En outre, - et c'est un point qui a, jusqu'à présent pu retenir l'attention des observateurs l'échinococcose aggrave sensiblement le polyparasitisme par Fasciola, Paramphistomes et «Strongles ».

- Sanitaire : Echinococcus polymorphus est capable d'infester non seulement les ruminants, mais encore l'homme. Malheureusement, on ne possède encore aucun renseignement sur l'incidence de l'échinococcose humaine dans cette partie -de l'Arussi.

\section{CONCLUSIONS}

Le parasitisme des zébus adultes de la région de Kofélé (Arussi) présente "grosso modo" les mêmes caractères que partout ailleurs en Ethiopie.

1. On note, dans 56 p. 100 des cas, l'existence d'un polyparasitisme à base de Trématodes hépatiques (Fasciola hepatica et Fasciola gigantica) ou gastriques (Paramphistomum daubneyi et Cotylophoron cotylophorum) et de divers « Strongles » gastro-intestinaux (Haemonchus contortus, Cooperia punctata, Cooperia pectinata, Trichostrongylus sp., Nematodirus sp., Bunostomum phlebotomum et Bosicola radiatum).

Sur 20 p. 100 de l'ensemble des animaux examinés, il revêt un caractère de haute gravité.

2. L'échinococcose est extrêmement répandue. Le pourcentage d'animaux infestés dépasse 
50 p. 100 avec, soit des localisations uniques, soit des localisations à plusieurs organes.

Les cas d'hydatidose pulmonaire sont plus nombreux que les cas d'échinococcose hépatique. La dégénérescence caséo-calcaire touche surtout les kystes hépatiques. Dans un certain nombre de cas, l'échinococcose se manifeste par des signes pulmonaires et par un amaigrissement brutal et marqué, pouvant aboutir à la mort.

3. La ladrerie à cysticerque inerme, Cysticercus bovis, sévit au taux de 14,6 p. 100.

L'importance de la cysticercose et de l'échinococcose bovine dans cette région de l'Ethiopie mérite d'être soulignée et, dans le cadre des projets de lutte contre ces zoonoses, la zone de Kofélé pourrait, éventuellement, servir de «zone pilote $»$.

\section{REMERCIEMENTS}

Nous tenons à remercier vivement Monsieur le Directeur de la "Quarantaine », ainsi que tout son personnel, qui nous ont permis de réaliser cette enquête dans les conditions les meilleures.

Nos remerciements iront également à Monsieur l'Inspecteur Général Desrotour, à Messieurs Grateau, Faye et Guidot pour toute l'aide qu'ils nous ont apportée.

\section{SUMMARY}

\section{Veterinary parasitology survey of adult cattle} in Kofele, Arussi province, Ethiopia

During winter 1976, investigations into parasitism of 85 zebu cattle of Kofélé (South of Arussi, Ethiopia) killed in Awassa (Sidamo) indicates the presence in the digestive tract of worms belonging to two groups :

- Trematodes (Paramphistomum daubneyi and Cotylophoron cotylophorum in duodenum and rumen) Fasciola hepatica and Fasciola gigantica in bile ducts).

- Nematodes (Haemonchus contortus, Cooperia punctata, Cooperia pectinata, Trichostrongylus sp., Nematodirus sp., Bosicola radiatum, Bunostomum phlebotomum) in the abomasum and in the gut.

Frequently, both Trematodes and Nematodes occur together in the same animal and this polyparasitism has serious harmful effects in twenty per cent of cases.

As in another parts of Ethiopia, two zoonoses are particularly important : Cysticercosis due to Cysticercus bovis with a rate of infection of 14,6 p. 100 and larval echinococcosis which affects more than 50 p. 100 of animals. In some cases, pulmonary hydatidosis may cause death.

\section{RESUMEN}

Inventario parasitologico de Ethiopia.

Helmintos de los cebues adultos de la región de Kofélé (Arussi, Etiopia)

Los autores dan la lista de los helmintos recogidos durante la autopsia de un grupo de cebues adultos de la región de Kofélé (sur de la provincia de Arussi, Etiopia).

Observan, en el tracto digestivo y en el higado, la presencia de numerosos Trematodos y Nematodos frecuentemente asociados entre ellos ; lo que representa un poliparasitismo que es particularmente grave en unos 20 p. 100 de los casos.

Por otro lado, encuentran igualmente las dos principales zoonosis tan distrıbuidas en Etiopia : la cisticercosıs (Cysticercus bovis) con una tasa de infestación de 14,6 p. 100 y la equinococosis (Echinococcus polymorphus) que ataca más de 50 p. 100 de los animales autopsiados y a veces provoca su muerte por caquexia.

\section{BIBLIOGRAPHIE}

1. BERGEON (P.). Veterinary parasitology survey. Rep. to the Government of Ethiopia. Rome, F. A. O., 1968,38 p. (n० T. A. 2458).

2. BERGEON (P.), LAURENT (M.). Différence entre la morphologie testiculaire de Fasciola hepatica et de
Fasciola gigantica. Rev. Elev. Méd. vét. Pays trop., 1970, 23 (2) : 223-227.

3. DAYNES (P.), GRABER (M.). Principales helminthoses des animaux domestiques d'Ethiopie. Rev. Elev. Méd. vét. Pays trop., 1974, 27 (3) : 301-306. 
4. DINNIK (J. A.). Paramphistomum daubneyi sp. nov. from cattle and its snail host in Kenya. Parasitology, 1962, 52 (1/2) : 143-151.

5. EUZEBY (J.). Diagnostic expérimental des helminthoses animales. Paris, Vigot frères, 1958.

6. GRABER (M.). Helminthes et helminthiases des animaux domestiques et sauvages d'Ethiopie. T. 1. Maisons-Alfort, I. E. M. V. T., 1973, 201 p. et Bull. Anim. Hith. Prod. Afr., 1975, 23 (1) : 57-85.

7. GRABER (M.). Helminthes et helminthiases des animaux domestiques et sauvages d'Ethiopie. T. 2. Maisons-Alfort, I. E. M. V. T., 1976, 206 p.

8. GRABER (M.), DAYNES (P.). Mollusques vecteurs de trématodoses humaines et animales en
Ethiopie. Rev. Elev. Méd. vét. Pays trop., 1974, 27 (3) : 307-322.

9. NASMARK (K. E.). A revision of the Trematode family Paramphistomidae. Inaug. Dissert. Zool. Bidrag. fran Uppsala, 1937, 16: 301-566.

10. PERSSON (I.), PERSSON (L.). Minor investigations on parasites of domestic animals in Ethiopia. Svensk Veterinartidn., 1970, $22: 19$.

11. PETITCLERC (M.). Awash (Ethiopie) : collecte d'informations sur la pathologie des animaux sauvages. Maisons-Alfort, I. E, M. V. T., 1977, 53 p.

12. VAN STRYDONCK (D). Contribution à l'étude de l'anatomie, de la morphologie et de la systématique des Paramphistomidae africains (Plathelminthes: Trematoda) Annls. Mus. r. Afr. cent., Sér. 8, 1970 (183) $56 \mathrm{p}$. 